\title{
QUE POURRA-T-ON DEDUIRE DES MESURES DE DISTANCE TERRE-LUNE PAR LASER?
}

\author{
J. KOVALEVSK Y \\ Observatoire de Meudon, France
}

\begin{abstract}
Résumé. Bien qu'il existe plusieurs stations de télémétrie de la lune par laser, une seule est tout-à-fait opérationnelle: celle de l'Observatoire de McDonald qui donne des erreurs internes d'observation inférieures à $15 \mathrm{~cm}$.

L'interprétation des données fait intervenir un grand nombre de paramètres se rapportant à la terre et à la lune et qui sont données.

Le laser-lune est particulièrement adapté pour déterminer les paramètres relatifs à la lune et, avec les futurs lasers de précision 2 à $3 \mathrm{~cm}$, on peut espérer que cette précision se retrouvera dans la détermination de ces paramètres.

La détermination probable du demi grand axe à $1 \mathrm{~cm}$ près en quelques mois, constituerait une nouvelle méthode d'évaluer la partie non conservative du mouvement de la lune. On peut s'attendre à une précision analogue pour la rotation de la lune. La situation pour les paramètres relatifs à la terre (rotation de la terre et mouvement du pôle) n'est pas aussi bonne à cause d'une présentation géométrique plus faible du problème et l'absence d'observations pendant une semaine par mois.

Néanmoins, cela donnera un contrôle externe très utile des résultats obtenus par les méthodes concurrentes (radio-interférométrie, satellites laser et radio).
\end{abstract}

\begin{abstract}
Although several lunar laser ranging stations exist, only one is now fully operational: the McDonald station with internal observational errors of less than $15 \mathrm{~cm}$. The interpretation of the data involves a great number of parameters relative to the Earth and the Moon which are listed. .

The lunar laser is particularly fit for those parameters that pertain to the Moon, and with future lasers accurate to 2 or $3 \mathrm{~cm}$, it may be expected that this accuracy will be projected into these parameters. The probable determination of the semi-major axis to $1 \mathrm{~cm}$ accuracy for a few months mean would imply a new means of determining the non conservative part of the motion of the Moon. A similar precision is to be expected for the rotation of the Moon. The situation for the Earth parameters (Earth rotation and polar motion) is not so good, because of a rather weak geometry of the problem and the monthly one week gap in the observations. Nevertheless, it will give a very useful external check on other competing methods (radio-interferometry, laser or radio-satellites).
\end{abstract}

C'est le ler août 1969 que la première distance entre un instrument terrestre et des cataphotes déposés sur la lune a été mesurée à l'observatoire Lick. Depuis, de nombreuses mesures analogues ont été faites, en grande majorité à l'observatoire de McDonald, mais aussi par d'autres équipes américaines, soviétiques, françaises et et japonaises.

$\mathrm{Au}$ total trois réflecteurs ont été déposés par les vols Apollo 11, 14 et 15, tandis que deux ensembles de réflecteurs français étaient montés sur les deux Lunakhod soviétiques.

Au cours de ces quatre années, ces instruments ont subi de nombreuses améliorations, tandis que de nouveaux lasers-lune sont en installation, le plus important étant celui que le groupe U.S. Lure va installer à la fin de 1973 aux îles Hawai.

En fait, actuellement, toutes les stations sauf une - celle de l'observatoire de McDonald - en sont à une phase d'expérimentation des techniques. C'est pourquoi, on 
ne peut que se fier aux résultats de cet observatoire pour connaître l'état actuel de la technique en ce domaine.

Le laser est un laser à rubis travaillant à 6943 ångströms avec une énergie de 3 joules, pour un tir toutes les 3 secondes avec une divergence angulaire de 4'. On utilise le télescope de 2,70 m de McDonald. La durée d'une impulsion est d'environ 4 nanosecondes, tandis que la calibration est maintenant faite avec une précision globale de $0,4 \mathrm{~ns}$ soit, en distance, $6 \mathrm{~cm}$. Le bruit interne des observations, qui est de $1 \mathrm{~ns}$ permet d'avoir actuellement des valeurs moyennes de la distance terre-lune à environ $15 \mathrm{~cm}$ prés.

L'interprétation de ces mesures se heurte à de nombreuses difficultés théoriques et pratiques qui sont loin d'être toutes résolues. Si on appelle $T$ et $L$ les centres de masse respectivement de la terre et de la lune, par $O$ la station d'observation et par $R$ le réflecteur sur la lune, l'observation étant faite entre les instants $t$ (instant de tir) et $t+\Delta t$ (instant de réception), et en appelant $t^{\prime}=t+\Delta t / 2$, on a avec une approximation surabondante

$$
\begin{aligned}
& \mathbf{O R}\left(t^{\prime}\right)=-\mathbf{T O}\left(t^{\prime}\right)+\mathbf{T L}\left(t^{\prime}\right)+\mathbf{L R}\left(t^{\prime}\right) \\
& \text { et on mesure en fait la distance } D=\left|\mathbf{O R}\left(t^{\prime}\right)\right|
\end{aligned}
$$

En analysant chacun des trois vecteurs composant $\mathbf{O R}$, on constate qu'un nombre impressionant de phénomènes entrent dans l'interprétation de la distance $D$, même une fois corrigée des effets instrumentaux (calibration) ou des effets de propagation (réfraction).

Tous ces vecteurs varient par suite de causes très diverses et, par ailleurs, ils doivent être tous repérés dans un système de référence unique, inertiel, par rapport auquel s'applique la théorie du mouvement de la lune.

$1^{\circ}$. Vecteur OT. Ce vecteur dépend:

- de la position de l'observatoire, donc de ses coordonnées géodésiques par rapport à un système de référence mondial.

- des mouvements de cet observatoire dû aux marées terrestres et, éventuellement, aux dérives continentales.

- de la position de ce système de référence par rapport à un système inertiel, donc des divers paramètres décrivant le mouvement du pôle, la rotation de la terre (TU1) ainsi que la précession et la nutation.

$2^{\circ}$ Vecteur TL. Ce vecteur représente le mouvement du centre de gravité de la lune et dépend donc:

- des six paramètres orbitaux ou conditions initiales du mouvement de la lune.

- des paramètres physiques qui déterminent les forces gravitationnelles subies par la lune: masses de la terre et de la lune, éléments de l'orbite de la terre, masses et éléments orbitaux des planètes, grandeur des perturbations des orbites de la terre et des planètes, coefficients principaux du potentiel de la terre.

- des paramètres physiques décrivant l'échange d'énergie dans le système terre-lune due à l'existence de la dissipation par le phénomène de marées et entraînant en particulier un terme quadratique dans la longitude de la lune et une augmenta- 
tion séculaire du demi-grand axe de son orbite.

$3^{\circ}$ Vecteur LR. Ce vecteur dépend:

- de la position du réflecteur dans un système de référence sélénodésique.

- des paramètres de la rotation de la lune qui dépendent eux mêmes des moments principaux d'inertie de la lune ainsi que d'autres moments d'ordre supérieur:

- du paramètre définissant la libration libre de la lune.

De nombreuses études théoriques ont été faites sur ce que l'on pourra effectivement déterminer par cette méthode. En effet, il n'est pas impossible, même s'il y a plusieurs stations terrestres et plusieurs cataphotes sur la lune de déterminer l'ensemble des paramètres dont dépend chaque mesure.

Il ne semble pas qu'il y ait, d'ailleurs, accord général sur ce que l'on peut potentiellement espérer. Une analyse de cette sorte ne peut pas être faite indépendamment d'une analyse parallèle des possibilités offertes par d'autres techniques, compte tenu de leur développement probable et de la couverture spatiale ou temporelle envisagée.

Il y a deux domaines, pour lesquels les laser-lune restent sans concurrents : ce sont ceux qui concernent les vecteurs TL et LR. En supposant, pour sérier les problèmes, que l'on puisse connaître indépendamment de façon parfaite, le vecteur OT, la mesure avec une précision de l'ordre de $15 \mathrm{~cm}$ d'une composante de TL + LR nous

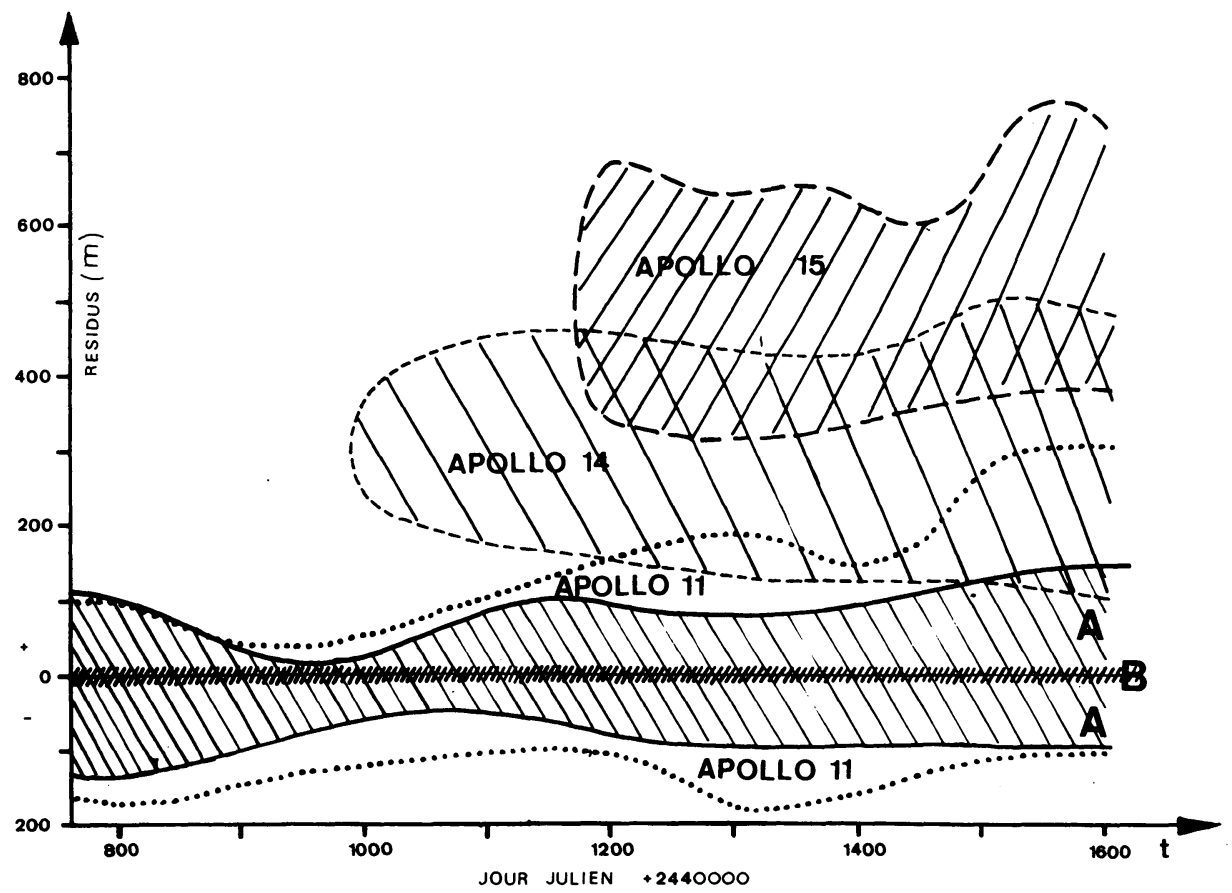

Fig. 1. Ordre de grandeurs des résidus de réduction des observations de laser-lune à McDonald (d'après Bender et al., 1973). Les régions notées Apollo 11, 14 et 15 indiquent les ordres de grandeur des résidus par rapport à des éphémérides indépendantes des observations. La région $\mathrm{A}$ est celle que l'on obtient pour les trois réflecteurs après correction des positions des réflecteurs et de l'observatoire. La région $\mathrm{B}$ $(<10 \mathrm{~m})$ indique où se trouvent les résidus après amélioration des paramètres orbitaux. 
permet d'atteindre avec une précision comparable les paramètres de rotation de la lune et ceux qui sont relatifs au mouvement orbital.

En ce qui concerne ce dernier, on peut, s'il n'y a pas d'effet systématique inconnu, espérer arriver à définir à $1 \mathrm{~cm}$ près le demi-grand axe moyenné sur plusieurs mois, ce qui rendra mesurable l'effet indirect de marées sur la lune estimé à environ 3 centimètres par an (d'après la formule de Spencer Jones). Le gain général par rapport à la connaissance de l'orbite de la lune avant l'ère spatiale (d'après les Improved Lunar Ephemeris) serait donc - en distance - de l'ordre d'un facteur $10^{-4}$ sur la précision. La figure 1 montre l'amélioration apportée dans la représentation des observations par l'amélioration de certains paramètres (position des réflecteurs et du laser, éléments orbitaux, etc....). L'amélioration des éphémérides correspondantes est d'au moins un ordre de grandeur.

Pour ce type d'études, il ne semble pas qu'il y ait de technique concurrente. Notons cependant que la précision relative avec laquelle on peut déduire la longitude de la lune (relativement à des repères uniquement terrestres) par la méthode laser est plus faible que la distance. Avec 2 stations à 6000 kilomètres et une précision de mesure de $15 \mathrm{~cm}$, on peut obtenir les éléments angulaires du réflecteur à environ 0.01 près par rapport à une base terrestre. A cette erreur, il faut ajouter les erreurs de passage au système de référence céleste. Ces dernières ne sont éliminées que par les méthodes classiques malheureusement insuffisamment précises de rattachement de la lune aux étoiles (observations d'occultations ou photographie sur fond d'étoiles) et surtout par la radio-interférométrie à longue base entre une source artificielle sur la lune et une radio-source naturelle. C'est cette dernière technique qui, seule, peut concurrencer le laser-lune dans un avenir lointain. Cependant sa mise en oeuvre est extrêmement lourde et difficile; il paraît peu probable que des observations de routine puissent être entreprises par cette technique. Aussi conclurons nous que le laser-lune a d'ores et déjà les capabilités d'accroître, jusqu'à des précisions de l'ordre du centimètre, notre connaissance du mouvement orbital de la lune avec les conséquences géophysiques sur l'évolution du système terre-lune que cela implique.

Le mouvement de rotation de la lune est actuellement mal connu. Les résidus des observations actuelles, au niveau de 5 à 10 mètres, sont essentiellement dus à une mauvaise théorie de la rotation de la lune. Il y a donc ici un gain possible de deux ou trois ordres de grandeur. Il est difficile à dire si un tel gain est suffisant pour atteindre des propriétés physiques de l'intérieur de la lune, mais cela semble peu probable. De toute façon cette connaissance est intrinsèquement utile et la méthode des observations à l'aide de laser lune est la seule qui soit adéquate et rentable. La seule technique concurrente serait celle du Doppler différentiel (improprement appelée radiointerférométrie différentielle). Shapiro a obtenu des résultats préliminaires très prometteurs, mais la lourdeur de la mise en oeuvre de cette technique est telle que la méthode du laser-lune apparaît comme étant meilleure et plus certaine d'être employée de façon suivie.

La question des possibilités offertes par le laser-lune pour déterminer les paramètres de variation du vecteur OT est plus délicate à traiter. Les études faites à ce sujet 
montrent que la précision du laser se répercute de façon assez dégradée sur les éléments relatifs à la rotation de la terre. On arrive, en gros à des précisions journalières de l'ordre de quelques décimètres à partir d'observations à $15 \mathrm{~cm}$ près. La difficulté essentielle est que le caractère aléatoire de ces mouvements interdit de cumuler les observations trop longtemps, contrairement à ce qu'on peut faire pour les mouvements relatifs à la lune. Il s'en suit donc que, même s'il y a une dizaine de lasers-lune répartis sur la surface du globe, le pouvoir séparateur spatio-temporel de cette méthode reste limité. Il est à comparer avec les possibilités d'ores et déjà atteintes pour le mouvement du pôle par un système peu précis de poursuite radio des satellites Transit. On atteint maintenant $40 \mathrm{~cm}$ pour 2 jours alors que, potentiellement, il y a au moins deux ordres de grandeur à gagner en améliorant la technologie du système. Les préétudes relatives à l'observation de petits satellites sphériques à 3000 kilomètres (LAGEOS) montrent qu'avec des lasers de même qualité que les actuels laser-lune (15 centimètres), on pourra obtenir TU1 et le mouvement du pôle avec beaucoup plus de finesse, simplement parce que, à couverture instrumentale égale, les conditions géométriques sont meilleures. Par ailleurs, l'absence d'observations de la lune pendant la semaine de la nouvelle lune est un grave inconvénient pour des études fines de la rotation de la terre et du mouvement du pôle qui sont nécessaires pour étudier leurs variations brusques, et les corréler avec d'autres phénomènes géophysiques.

En ce qui concerne la détermination des relations entre les systèmes de référence, cette technique ne pouvant pas atteindre les axes stellaires, son efficacité est assez faible, bien qu'il y ait un certain intérêt à relier la position de la lune à des repères terrestres, comme nous l'avons vu plus haut. Mais, de façon générale, les méthodes de radio-interférométrie à longue base sont beaucoup plus efficaces pour ce problème.

Pour nous résumer, nous disons donc que, avec la précision actuelle du laser-lune de l'observatoire de McDonald, le laser-lune permet un fantastique gain en précision pour tous les mouvements de la lune et semble être la technique la plus simple à mettre en oeuvre et la plus efficace. En revanche, pour les paramètres terrestres, elle a des concurrents mieux placés et ne peut prétendre qu'à un rôle d'appoint ou, ce qui est très utile, de contrôle des autres méthodes pour la discussion des erreurs systématiques.

Mais on peut objecter que la précision des laser-lune va augmenter. En effet des lasers donnant 3 centimètres sont en construction. Les limitations des possibilités de cette technique proviennent d'une part des dimensions des cataphotes lunaires dont l'orientation relative à la terre varie avec la libration et de la difficulté de modéliser à mieux que 1 centimètre près l'effet de la réfraction atmosphérique. En estimant à 2 centimètres cette limite, on voit que cela renforce encore nos conclusions sur l'efficacité de la méthode pour l'étude du mouvement de la lune. Mais cela ne modifie pas non plus les conclusions relatives à la terre, car les mêmes améliorations seront apportées aux lasers de télémètre de satellites; d'autre part, comme nous l'avons dit, il y a encore une grande marge de possibilités d'améliorations des systèmes radioélectriques, et enfin, l'interférométrie à longue base aura un grand rôle à jouer dans la recherche du système de référence. 
En conclusion, nous dirons que, dans l'avenir, le laser-lune est capable de fournir une précision centimétrique dans l'étude du mouvement de la lune, notamment dans la détermination des termes non gravitationnels et il est compétitif, sans être le meilleur système possible pour les études relatives aux mouvements et aux déformations de la terre.

\section{Bibliographie}

Une bibliographie très complète a été donnée par Bender, P. L., Curie, D. G., et al.: 1973, Science 182, 229.

\section{DISCUSSION}

Van Herk: Are the effects of the Earth-tides known so well that they can be corrected for?

Mulholland: The modellability of solid Earth tides depends strongly on location. Near ocean margins, the situation is not good, but a far inland site such as McDonald Observatory behaves very closely in accord with the presently available theory.

Kovalevsky: This is why, for near oceanic sites like those in Europe, it is necessary to monitor the actual motions of the local crust by gravimeters and horizontal pendulums.

Mulholland: It is worth pointing out the significance of a determination of the secular variation of the lunar semi-axis. Kovalevsky's value of $3 \mathrm{~cm} \mathrm{yr}^{-1}$ assumed a specific value of the secular acceleration in longitude. The conventional value of $11^{\prime \prime} / \mathrm{cy}$, determined by Spencer-Jones some 35 years ago, is apparently still unexplainably large for geophysicists. In recent years, four different investigations have resulted in derived values $100 \%$ larger than Spencer-Jones' value. Thus, a direct determination of $\mathrm{d} a / \mathrm{d} t$ should be very important.

It is quite true that the lunar problems will dominate the data analysis for some time to come. For example, Williams and others have shown that the higher moments of the lunar figure produce quite sensible effects on the libration, sufficiently so that one may expect that certain 3rd order gravity harmonics will likely soon be best determined from laser ranging.

Murray: Can the vector OT be determined adequately from satellite ranging?

Kovalevsky: Not fully. I believe that this will be possible as far as mean position of $T$ and motion of the pole and rotation of the Earth are concerned, using satellites like LAGEOS for instance. The local tidal effects should be measured on the spot with gravimeters and horizontal pendulums.

Murray: Will it ultimately be possible to determine the mean orbit of the moon and hence the ecliptic, relative to the geodetic frame? If so, we could measure the precession from purely distance measurements.

Kovalevsky: I have not given this problem sufficient thought. I believe that it is theoretically possible, but I cannot commit myself on the eventual accuracy of the determination. 